
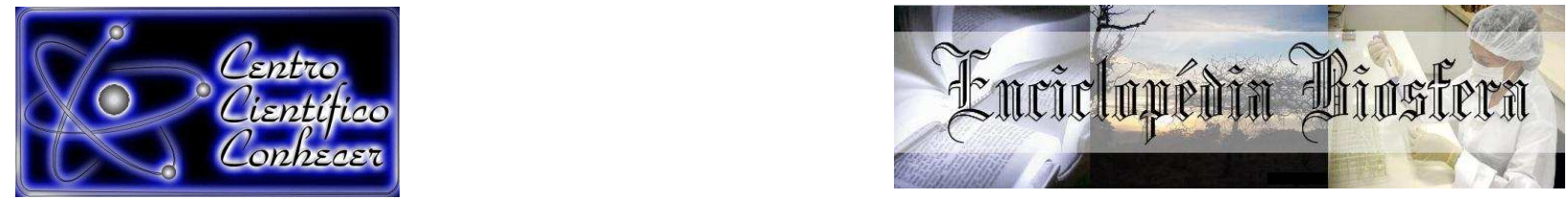

\title{
COLONIZAÇÃO NASAL POR ESTAFILOCOCOS COAGULASE NEGATIVA EM PROFISSIONAIS DE UMA INSTITUIÇÃO DE SAÚDE NA REGIÃO NORTE DO ESPÍRITO SANTO
}

\author{
Josiane Correia Magy dos Santos ${ }^{1}$, Mirella Gama Matos ${ }^{2}$, Débora Barreto Teresa \\ Gradella $^{3}$
}

1- Farmacêutica do Laboratório de Análises Clínicas Labol Exames, Barra de São Francisco, ES - Brasil

2- Farmacêutica do Hospital Unimed, Linhares, ES - Brasil

3- Professora Doutora do Departamento de Ciências da Saúde da Universidade Federal do Espírito Santo (debora.gradella@ufes.br), São Mateus, ES Brasil.

Recebido em: 08/09/2015 - Aprovado em: 14/11/2015 - Publicado em: 01/12/2015 DOI: http://dx.doi.org/10.18677/Enciclopedia_Biosfera_2015_039

\begin{abstract}
RESUMO
As bactérias do gênero Staphylococcus apresentam-se como cocos Gram-positivos, agrupados principalmente em cachos e são encontradas na microbiota normal humana (pele e mucosas). São classificadas pela capacidade de coagular o plasma em coagulase positivas ou coagulase negativas. Os estafilococos coagulase negativa $(E C N)$ tornaram-se relevantes na clínica após a identificação desses microrganismos em infecções. $O$ aparecimento de cepas resistentes a antimicrobianos em ambiente hospitalar aumenta a preocupação em relação a esses microrganismos. O presente estudo teve por objetivo avaliar a frequência de colonização nasal por ECN e seu perfil de resistência à meticilina em profissionais de uma Instituição de Saúde na região norte do Espírito Santo. As amostras foram coletadas da nasofaringe dos voluntários, isoladas em Ágar Salgado Manitol e identificadas pela técnica de coloração de Gram, prova da catalase e teste da coagulase livre. O perfil de resistência a meticilina foi avaliado pela suscetibilidade do microrganismo frente ao disco de oxacilina. Das 38 amostras coletadas, isolou-se em $31(81,6 \%)$ o ECN. De 30 amostras de ECN testadas para avaliar a sensibilidade a oxacilina, $23(76,7 \%)$ apresentaram resistência. Verificou-se que os profissionais em contato direto com os pacientes possuem com maior frequência ECN resistentes. Portanto, considera-se importante o cuidado com a disseminação de estirpes resistentes de microrganismos por profissionais em ambiente hospitalar, evitando-se ocasionar maiores prejuízos para a saúde dos pacientes e maiores gastos para os serviços de saúde.
\end{abstract}

PALAVRAS-CHAVE: Estafilococos coagulase negativa, perfil de resistência, profissionais de saúde. 


\title{
NASAL COLONIZATION FOR COAGULASE NEGATIVE STAPHYLOCOCCI IN PROFESSIONALS OF A HEALTH INSTITUTION IN THE REGION NORTHERN OF THE ESPIRITO SANTO
}

\begin{abstract}
The bacteria of the genus Staphylococcus present as Gram-positive cocci, grouped in clusters and are mainly found in normal human flora (skin and mucosa). They are classified by the ability to clot plasma coagulase positive or negative coagulase. The coagulase-negative Staphylococci (CNS) have become relevant in the clinic after the identification of these microorganisms in infections. The emergence of antimicrobialresistant strains in hospitals raises concerns regarding these microorganisms. This study aimed to evaluate the frequency of nasal colonization by CNS and its resistance profile methicillin professionals of a health institution in the region northern of the Espirito Santo. Samples were collected from the nasopharynx of volunteers, isolated Mannitol Salt Agar and identified by Gram staining technique, the catalase test and free coagulase. The methicillin resistance profile was evaluated by susceptibility of the organism against the oxacillin disk. Of the 38 samples collected, isolated in $31(81.6 \%)$ the CNS. Of 30 samples CNS assayed for sensitivity to oxacillin, $23(76.7 \%)$ showed resistance. It was found that professionals in direct contact with patients have more frequently resistant CNS. Therefore, it is considered important to the care of the spread of resistant strains of microorganisms by professionals in a hospital setting, avoiding causing major damage to the health of patients and higher expenses for health services.
\end{abstract}

KEYWORDS: Coagulase negative Staphylococci, Health professionals, Resistance profile.

\section{INTRODUÇÃO}

Os cocos Gram positivos são uma coleção heterogênea de bactérias. O nome do gênero Staphylococcus refere-se ao fato de que as células destes cocos crescem com um perfil que se assemelha a cachos de uvas. São catalase-positivos, não produzem esporos e a maioria dos estafilococos mede de 0,5 a 1,5 $\mu \mathrm{m}$ de diâmetro, são capazes de crescer em meios contendo alta concentração de sal e temperaturas que variam de $18^{\circ} \mathrm{C}$ a $40^{\circ} \mathrm{C}$ (MURRAY et al., 2013).

Esse gênero compreende mais de 40 espécies diferentes sendo divididos de acordo com a produção da enzima coagulase, em coagulase positivo e coagulase negativo. Entre os positivos, destaca-se o Staphylococcus aureus e Staphylococcus pseudintermedius, e entre Estafilococos coagulase negativos (ECN) pode-se destacar o Staphylococcus epidermidis e Staphylococcus haemolyticus. As espécies desse gênero fazem parte da microbiota normal humana, habitando pele e mucosas (SHORE \& COLEMAN, 2013).

Até a década de 70 , os ECN eram vistos somente como contaminantes de pouca importância clínica e não como patógenos causadores de infecções hospitalares. Atualmente tornou-se uma causa frequente destas infecções (CORDEIRO, 2007), sendo comumente associados a infecções oportunistas (SHORE \& COLEMAN, 2013). De acordo com OLAECHEA e colaboradores (2011) as bacteremias causadas por ECN constituem uma das infecções hospitalares mais comuns em pacientes de unidade de tratamento intensivo.

Em geral, é a habilidade de se aderir e permanecer em superfícies de dispositivos médicos implantados, formando um biofilme, que se destaca nesse grupo de bactérias, já que a maioria dos ECN não produz muitas toxinas e 
exoenzimas que podem ocasionar danos em tecidos (KEIM, 2005). Os Staphylococcus apresentam elevada capacidade de adquirir resistência a diversos antimicrobianos, isso é especialmente evidenciado no ambiente hospitalar, onde esses medicamentos são amplamente utilizados exercendo uma pressão seletiva sobre estes microrganismos (CORDEIRO, 2007).

Devido à necessidade do uso de antimicrobianos para o tratamento de infecções, o surgimento de resistência é inevitável. Porém, o uso racional pode diminuir o aparecimento de estirpes multirresistentes (VIANA et al., 2011). A resistência dos estafilococos à meticilina é codificada pelo gene MecA que promove a modificação da enzima alvo do antimicrobiano, diminuindo a afinidade dessa enzima pelo antibiótico, tornando o microrganismo resistente (SOUZA, 2009). Esses ECN resistentes estão sendo atualmente relacionados à transmissão de genes de resistência para o Staphylococcus aureus (ALMEIDA, et al., 2014).

Isolados bacterianos multirresistentes são constantemente disseminados no ambiente hospitalar e apresentam como principal fonte de disseminação os profissionais de saúde, atribuindo também a estes a importância em surtos (RABELO, 2012). Segundo CORDEIRO (2007), as infecções nosocomiais serão controladas principalmente quando medidas simples como a lavagem frequente das mãos, e o uso racional de antimicrobianos forem adotadas.

Importante se torna a implantação da educação continuada entre os profissionais que atuam em ambiente hospitalar quanto às medidas de controle de infecção, bem como, uma vigilância bacteriológica entre estes (ALMEIDA, et al., 2014). Neste contexto, este estudo teve por objetivos pesquisar a presença de ECN em amostras de nasofaringe dos funcionários de um hospital do município de São Mateus-ES, verificar a resistência das amostras de ECN frente ao antimicrobiano oxacilina e correlacionar a presença de ECN e ECN resistentes à meticilina, com as variáveis sexo, categoria profissional e uso frequente de antibióticos.

\section{MATERIAL E MÉTODOS}

\section{Casuística}

O estudo foi realizado com profissionais de vários setores de uma Instituição de Saúde particular e de baixa complexidade de um município da região norte do estado do Espírito Santo durante o período de março a julho de 2012.

Os profissionais da Instituição foram convidados a participar da pesquisa e os que aceitaram assinaram o Termo de Consentimento Livre e Esclarecido. Foram excluídos os que estavam em antibioticoterapia e aqueles que fizeram uso de antibióticos nos últimos 30 dias. Foi aplicado ainda um questionário que teve por objetivo coletar informações sobre idade, sexo, setor de trabalho, categoria profissional, turno de trabalho, carga-horária de trabalho, uso de equipamentos de proteção individual e uso de antimicrobianos sem prescrição médica.

Este projeto foi submetido ao Comitê de Ética em Pesquisa e aprovado sob o parecer 33/2011 CEPCEUNES/UFES.

\section{Coleta e processamento do material clínico}

As amostras da nasofaringe dos profissionais foram coletadas por meio de "swab" que foi inserido por 10 a 15 segundos dentro das narinas, realizando movimentos rotatórios para atingir a mucosa nasofaríngea. Após a coleta os "swabs" foram colocados em tubo contendo caldo $\mathrm{BHI}$ e encaminhados ao Laboratório de Microbiologia do CEUNES, onde o material foi incubado por 12 horas à $37^{\circ} \mathrm{C}$. 


\section{Isolamento e identificação}

Após incubação, uma alíquota dos tubos que apresentaram turvação foi semeada em meio Ágar Salgado Manitol que foi incubado por período de 24 a 48 horas a $37^{\circ} \mathrm{C}$. Posteriormente, as placas foram analisadas quanto ao crescimento de microrganismos.

Para caracterização e identificação dos ECN, foram utilizadas as técnicas de Coloração de Gram, Prova da Catalase e Teste da Coagulase Livre (OPLUSTIL et al., 2010).

\section{Teste de Suscetibilidade à Oxacilina}

Para avaliar o perfil de resistência à meticilina foi observada a suscetibilidade do microrganismo frente ao disco de oxacilina pelo método de difusão de disco. Este método consiste na utilização de discos de papel filtro impregnados com uma concentração padronizada de antimicrobiano.

Para a realização deste teste deve-se preparar um inóculo usando o método de suspensão direta de colônias. Onde são transferidas colônias do microrganismo a ser testado para um tubo contendo salina estéril até que se alcance turbidez semelhante a existente no tubo 0,5 da escala de McFarland. Posteriormente, essa suspensão é semeada com "swab" em três direções diferentes nas placas de ágar Mueller-Hinton e sobre a semeadura adiciona-se 0 disco de antimicrobiano (OPLUSTIL et al., 2010).

As placas são incubadas à temperatura de $37^{\circ} \mathrm{C}$ por 24 horas. Após o período de incubação, realiza-se a medida dos halos de inibição com o emprego de régua milimetrada, e o uso de valores de referência permite a classificação da amostra bacteriana como sensível $(\mathrm{S})$ e resistente $(\mathrm{R})$.

\section{Análise dos dados}

Os dados foram reunidos e organizados em tabelas utilizando os Programas Microsoft Word® e Excel® 2013.

\section{RESULTADOS E DISCUSSÃO}

\section{Caracterização da amostra}

Aceitaram participar do estudo 38 voluntários. A faixa etária variou de 18 a 61 anos com média de idade de 34 anos. Desses, 10 (26,3\%) profissionais foram do sexo masculino e $28(73,7 \%)$ do sexo feminino. Em um estudo realizado por CRUZ (2008) em um hospital universitário, buscando identificar a presença de $S$. aureus em funcionários, o sexo feminino também correspondeu a uma maior parte da amostra $(72,3 \%)$ o que poderia refletir a predominância do sexo feminino em serviços de saúde.

De acordo com a Tabela 1 pode ser observada a distribuição dos voluntários segundo algumas variáveis do estudo. Nota-se que dentre as categorias profissionais, a mais frequente foi de técnico em enfermagem (28,9\%). Ainda, a maioria dos profissionais (55,3\%) trabalha em ambiente hospitalar a cinco anos ou mais. Uma pequena porcentagem dos profissionais $(28,95 \%)$ trabalha em outra instituição de serviço de saúde. Quanto ao turno de trabalho, o integral, compreende o turno com maior número de profissionais $(78,9 \%)$. 
TABELA 1- Distribuição dos profissionais de uma Instituição de Saúde do Norte do Espírito Santo participantes da pesquisa, segundo as variáveis, categoria profissional, tempo de serviço, turno e vínculo de trabalho.

\begin{tabular}{|c|c|c|}
\hline Variável & & \\
\hline Categoria do trabalho & $\mathbf{N}$ & $\%$ \\
\hline Acadêmico de Medicina & 2 & 5,3 \\
\hline Atendente de Farmácia & 3 & 7,9 \\
\hline Auxiliar Administrativo & 6 & 15,8 \\
\hline Auxiliar de Enfermagem & 1 & 2,6 \\
\hline Auxiliar de Serviços Gerais* & 4 & 10,5 \\
\hline Enfermeiro & 1 & 2,6 \\
\hline Médico & 3 & 7,9 \\
\hline Recepcionista & 6 & 15,8 \\
\hline Técnico em Enfermagem & 11 & 28,9 \\
\hline Técnico em Radiologia & 1 & 2,6 \\
\hline Total & 38 & 100,0 \\
\hline $\begin{array}{l}\text { Tempo de atuação em amb } \\
\text { hospitalar }\end{array}$ & & \\
\hline Inferior a 5 anos & 17 & 44,7 \\
\hline 5 anos ou mais & 21 & 55,3 \\
\hline Total & 38 & 100,0 \\
\hline Turno de trabalho & & \\
\hline Matutino & 2 & 5,3 \\
\hline Vespertino & 1 & 2,6 \\
\hline Noturno & 3 & 7,9 \\
\hline Matutino e Vespertino & 2 & 5,3 \\
\hline Integral & 30 & 78,9 \\
\hline Total & 38 & 100,0 \\
\hline Trabalha em outra Instituiç & & \\
\hline Sim & 11 & 28,9 \\
\hline Não & 27 & 71,1 \\
\hline Total & 38 & 100,0 \\
\hline
\end{tabular}

N: número; \%: porcentagem

* Funcionários dos setores de limpeza e lavanderia.

Ao analisar a variável categoria profissional (Tabela 1), observa-se que a mesma é composta por profissionais que apresentam contato direto com o paciente e por profissionais que raramente o apresentarão, devido a atividade que exercem no ambiente hospitalar. Sendo assim, os profissionais que apresentam contato direto com os pacientes do hospital foram acadêmicos de medicina, médicos, enfermeiros, técnicos e auxiliares de enfermagem e técnico em radiologia, e os que não apresentam este contato foram os do setor administrativo e contábil, auxiliares de serviços gerais, recepcionistas e auxiliares de farmácia. Assim, os profissionais que participaram do presente estudo encontram-se distribuídos de forma idêntica em ENCICLOPÉDIA BIOSFERA, Centro Científico Conhecer - Goiânia, v.11 n.22; p.3232 
cada grupo, ou seja, 19 (50\%) profissionais mantêm contato direto com os pacientes e $19(50 \%)$ não apresentam este contato.

\section{Identificação dos ECN resistência antimicrobiana}

Após análise das amostras coletadas dos 38 participantes deste estudo, ECN foram identificados em 31 (81,6\%). Em estudo realizado por PEREIRA \& CUNHA (2009) com o objetivo de avaliar a colonização nasal por Staphylococcus spp. em alunos de enfermagem, $72,5 \%$ dos Staphylococcus isolados foram ECN. ALMEIDA e colaboradores (2014) isolaram Staphylococcus spp. em quase $90 \%$ das amostras da cavidade nasal de pacientes hospitalizados, sendo que a maioria foi de ECN (61\%). ABADI e colaboradores (2015) em um estudo com estudantes de 7 a 19 anos encontraram um total de $71,7 \%$ dos voluntários carreando ECN em suas narinas. RIBEIRO e colaboradores (2014), em estudo realizado com estudantes da Universidade de Fortaleza buscando identificar Staphylococcus aureus na cavidade nasal e nas mãos dos estudantes, verificaram uma porcentagem de $61,8 \%$ destes com ECN e 38,2\% com $S$. aureus.

Em outra pesquisa, MORAES e colaboradores (2013), pesquisaram a presença de ECN em materiais e superfícies de três unidades de terapia intensiva neonatal e infantil. Estes pesquisadores encontraram um total de $66,7 \%$ das superfícies e materiais pesquisados colonizados por ECN. Esse estudo é muito importante devido a capacidade de estes microrganismos causarem infecções em recém-nascidos e crianças, visto que os profissionais de saúde podem disseminar tais microrganismos entre os pacientes. Pesquisando microrganismos em jalecos de profissionais de um hospital, OLIVEIRA \& SILVA (2013) isolaram do total da amostra: Staphylococcus epidermidis (41\%), Staphylococcus hominis (23\%), Staphylococcus capitis (18\%), Staphylococcus haemolyticus (9\%) e Staphylococcus warneri (9\%).

Todos os $E C N$ isolados ( $n=30$ ) passaram pelo teste de resistência a oxacilina pelo método de difusão em disco. Após medida do halo foi possível verificar que amostras de sete profissionais (23,3\%) foram sensíveis e de $23(76,7 \%)$ apresentaram resistência ao antimicrobiano testado (Tabela 2). Percentuais de resistência menores foram obtidos por alguns autores. ROSA et al. (2009), em um estudo em que se pesquisava o ECN na saliva de profissionais de enfermagem encontraram $32 \%$ de resistência à oxacilina. Porcentagens ainda menores foram encontradas por PEREIRA \& CUNHA (2009), onde 16,2\% dos ECN isolados de amostras nasais de estudantes de enfermagem foram resistentes à oxacilina.

No presente estudo, a maior porcentagem de ECN resistentes à oxacilina foi encontrada entre os técnicos de enfermagem, porém estes compreenderam os profissionais em que mais se isolou os ECN (Tabela 2). Assim, não é possível afirmar que estes profissionais são os mais colonizados por ECN resistentes à oxacilina. Técnicos de enfermagem foram os profissionais mais colonizados por Staphylococcus spp. resistentes a meticilina em estudo realizado por RABELO (2012), representando $48,15 \%$ dentre as amostras positivas. 
TABELA 2- Distribuição dos portadores de ECN sensíveis e resistentes a oxacilina entre os profissionais de uma Instituição de Saúde do Norte do Espírito Santo, segundo as variáveis, sexo, idade, setor de trabalho, tempo de serviço, turno e vínculo de trabalho

\begin{tabular}{lcccc}
\hline Variável & ECN sensível & \multicolumn{2}{c}{ ECN resistente } \\
\hline Sexo & $\mathbf{N}$ & $\%$ & $\mathbf{N}$ & $\%$ \\
\hline Feminino & 6 & 85,7 & 17 & 73,9 \\
Masculino & 1 & 14,3 & 6 & 26,1 \\
\hline Total & 7 & 100,0 & 23 & 100,0 \\
\hline Categoria do trabalho & & & & \\
\hline Acadêmico de Medicina & 1 & 14,3 & 0 & 0,0 \\
Atendente de Farmácia & 0 & 0,0 & 2 & 8,7 \\
Auxiliar Administrativo & 3 & 42,9 & 2 & 8,7 \\
Auxiliar de Enfermagem & 0 & 0,0 & 1 & 4,3 \\
Auxiliar de Serviços Gerais & 1 & 14,3 & 3 & 13,0 \\
Médico & 0 & 0,0 & 3 & 13,0 \\
Recepcionista & 1 & 14,3 & 4 & 17,4 \\
Técnico em Enfermagem & 1 & 14,3 & 7 & 30,4 \\
Técnico em Radiologia & 0 & 0,0 & 1 & 4,3 \\
\hline Total & 7 & 100,0 & 23 & 100,0 \\
\hline Tempo de atuação em & & & & \\
ambiente hospitalar & & & 11 & 47,8 \\
\hline Inferior a 5 anos & 4 & 57,1 & 12 & 52,2 \\
\hline anos ou mais & 3 & 42,9 & 23 & 100,0 \\
\hline Total & 7 & 100,0 & &
\end{tabular}

$\mathrm{N}$ : número; \%: porcentagem

${ }^{*}$ A amostra de ECN de um participante da pesquisa foi perdida

Ao considerar como variável o contato ou não com o paciente, houve maior porcentagem de colonização de ECN resistentes em profissionais que mantem contato direto, enquanto que a maioria dos ECN sensíveis foi encontrada em profissionais que não apresentam esse contato (Tabela 3). Esta comparação é importante devido a possibilidade de transmissão de microrganismos resistentes, visto estes terem sido encontrados principalmente em profissionais que atuam diretamente com os pacientes.

TABELA 3- Distribuição dos portadores de ECN sensíveis e resistentes segundo contato com paciente e uso de antimicrobianos sem prescrição médica.

\begin{tabular}{lcccc}
\hline Variável & \multicolumn{2}{l}{ ECN sensível } & \multicolumn{2}{c}{ ECN resistente } \\
\hline Contato com o paciente & $\mathbf{N}$ & $\%$ & $\mathbf{N}$ & $\%$ \\
\hline Contato direto & 2 & 28,6 & 12 & 52,2 \\
Sem contato direto & 5 & 71,4 & 11 & 47,8 \\
\hline Total & 7 & 100,0 & 23 & 100,0 \\
\hline Uso de antimicrobianos & & & & \\
sem prescrição & & & & \\
\hline Frequentemente & 0 & 0,0 & 4 & 4,3 \\
Esporadicamente & 2 & 28,6 & 17 & 73,4 \\
Nunca & 5 & 71,4 & 1 & 4,3 \\
Não respondeu & 0 & 0,0 & 17 & 100,0 \\
\hline Total & 7 & 100,0 & 23 &
\end{tabular}

$\mathrm{N}:$ número; \%: porcentagem 
Quanto ao uso de antimicrobianos sem prescrição, a maior parte dos profissionais afirmou nunca utilizar. No entanto, $28,6 \%$ dos profissionais portadores de ECN sensível a oxacilina e $17,4 \%$ dos portadores de ECN resistentes relataram utilizar esporadicamente (Tabela 3).

A resistência bacteriana a antimicrobianos é inevitável e irreversível. Ocorre como uma consequência natural da célula bacteriana que possui habilidade de adaptar-se quando exposta aos antibióticos (SANTOS, 2004). Entretanto, o uso indiscriminado de antibióticos promove o aparecimento de estirpes multirresistentes tornando necessário utilizar antibióticos de maior espectro para o tratamento de infecções causadas por estes microrganismos (OLIVEIRA \& LIMA, 2010).

ROSA et al. (2009) encontraram resistência elevada de ECN à mupirocina. Este antimicrobiano é utilizado para descolonização de indivíduos portadores de Staphylococcus aureus resistente a oxacilina. Acredita-se que a resistência elevada dos ECN seja também decorrente da utilização deste antimicrobiano. Por isso, há a necessidade de se avaliar as políticas de controle de microrganismos multirresistentes adotadas em serviços de saúde. De acordo com OLIVEIRA \& DE PAULA (2012), a descolonização deve ser realizada apenas em pacientes de risco como aqueles internados em unidade de terapia intensiva, pacientes que permanecem internados por um longo período e pacientes cirúrgicos.

É imprescindível que os profissionais de saúde tomem conhecimento das graves consequências do uso indiscriminado de antibióticos (SANTOS, 2004). Bem como, se conscientizem quanto a importância de adotar medidas de assepsia e da relevância de estabelecer programas de educação continuada quanto às medidas de controle de infecção no ambiente em que atuam (ALMEIDA et al., 2014).

Dentre os profissionais portadores de $\operatorname{ECN}(n=30)$, foi considerado que 18 necessitam da utilização de Equipamentos de Proteção Individual (EPI's), pois estes atuam diretamente com os pacientes, manipulam medicamentos e realizam atividades que requerem proteção, como por exemplo, a limpeza do ambiente hospitalar. Destes profissionais que necessitam de EPI's, $17(94,4 \%)$ responderam que os utilizam, enquanto um (5,6\%) afirmou não utilizar. Cabe ressaltar, que o voluntário que afirmou não utilizar EPl's mantem contato direto com os pacientes, fato que chamou atenção, pois a utilização de EPI's é de extrema importância em meio hospitalar, principalmente para profissionais que estão relacionados ao cuidado com o paciente. Com relação à dificuldade apresentada pelos profissionais em utilizar EPI's, 7 (38,9\%) afirmaram que não há nada que impeça o uso, quatro $(22,2 \%)$ declararam achar desconfortável, três $(16,7 \%)$ disseram que os equipamentos não estão disponíveis nas unidades em quantidade suficiente e dois $(11,1 \%)$ disseram existir outros motivos, porém não os especificaram. Além disso, um $(5,5 \%)$ profissional relatou que o acesso é difícil e um $(5,5 \%)$ que não utiliza algumas vezes por esquecimento.

A utilização de EPI's reduz o risco de acidente ocupacional, bem como tornase uma conduta imprescindível para garantia de um tratamento sem risco de contaminação, tanto para o profissional quanto para o paciente. Por isso, torna-se importante a conscientização dos profissionais de saúde para que estes adotem efetivamente condutas e procedimentos que garantam a segurança (CARVALHO et al., 2009).

Em uma pesquisa sobre contaminação de jalecos de profissionais de saúde de um hospital universitário na cidade de Belo Horizonte, $76 \%$ relataram utilizar o jaleco somente no setor em que trabalha. Porém, $24 \%$ disseram utilizá-lo também em outras áreas do hospital como lanchonete, refeitório e área administrativa. $O$ fato 
de usar o jaleco fora da unidade é preocupante visto que esses profissionais podem estar disseminando microrganismos importantes no ambiente em que frequentam (OLIVEIRA \& SILVA, 2013).

\section{CONCLUSÃO}

De acordo com os resultados apresentados, pode-se concluir que $81,6 \%$ dos profissionais da Instituição estudada são colonizados por ECN e que destes $76,7 \%$ foram resistentes ao antimicrobiano oxacilina.

Observou-se que o ECN está presente em profissionais de vários setores dessa instituição, independente do contato destes com os pacientes. Nota-se assim, a presença do microrganismo em todo o ambiente hospitalar.

O elevado percentual de ECN é algo esperado devido estes microrganismos fazerem parte da microbiota normal, porém, a presença elevada de resistência entre os isolados $(76,7 \%)$ merece maior atenção, principalmente pela presença em profissionais que apresentam contato direto com o paciente $(52,2 \%)$, o que poderia facilitar a disseminação deste patógeno. A disseminação de estirpes resistentes por profissionais em ambiente hospitalar pode ocasionar maiores prejuízos para a saúde do paciente e maiores gastos para os serviços de saúde.

Assim, espera-se que após o conhecimento dos resultados do presente trabalho os profissionais tomem conhecimento sobre o seu estado de portador de microrganismos resistentes e que possam compreender melhor essa temática. Além disso, é importante que se estabeleçam programas de educação continuada, visando o conhecimento por parte de todos os funcionários das implicações dos microrganismos no ambiente hospitalar, da importância da lavagem e higienização das mãos, da utilização de equipamentos de proteção individual e do uso racional de antimicrobianos.

\section{REFERÊNCIAS}

ABADI, M. I. M.; MONIRI, R.; KHORSHIDI, A.; PIROOZMAND, A.; MOUSAVI, S. G. A.; DASTEHGOLI, K.; GHAZIKALAYEH, H. M. Molecular Characteristics of Nasal Carriage Methicillin-Resistant Coagulase Negative Staphylococci in School Students. Jundishapur Journal of Microbiology, v. 8, n. 6, 2015.

ALMEIDA, G. C. M.; LIMA, N. G. M.; SANTOS, M. M.; MELO, M. C. N.; LIMA, K. C. Colonização nasal por Staphylococcus sp. em pacientes internados. Acta Paulista de Enfermagem, v. 27, n. 3, p. 273-279, 2014.

CARVALHO, C. M. R. S.; MADEIRA, M. Z. A.; TAPETY, F. I.; ALVES, E. L. M.; MARTINS, M. C. C.; BRITO, J. N. P. O. Aspectos de bioseguridad relacionados al uso del jaleco por profesionales de salud: una revisión bibliográfica. Texto \& Contexto Enfermagem, v.18, n. 2, p. 355-60, 2009.

CORDEIRO, D. N. G. Significância clínica da presença de Staphylococcus coagulase-negativo isolados de recém-nascidos de uma unidade de terapia intensiva neonatal em Brasília-DF. 2007. Dissertação. Programa de pósgraduação em Medicina Tropical, Universidade de Brasília. Brasília, 2007.

CRUZ, E. D. A. Staphylococcus aureus e Staphylococcus aureus resistente à meticilina em trabalhadores de um hospital universitário: colonização e crenças em saúde. 2008. Tese (Doutorado em Enfermagem) - Programa de Interunidades de 
Doutoramento em Enfermagem dos campi de São Paulo e Ribeirão Preto da Universidade de São Paulo. Ribeirão Preto, 2008.

KEIM, L. S. Mapeamento dos estafilococos coagulase negativo no Hospital Universitário Antônio Pedro da Universidade Federal Fluminense, no período de 1998 a 2002. 2005. 133f. Dissertação (Mestrado em Ciências Médicas). Universidade Federal Fluminense. Niterói, 2005.

MORAES, C. L.; RIBEIRO, N. F. G.; COSTA, D. M.; FURLAN, V. G.; PALOS, M. A. P.; VASCONCELOS, L. S. N. O. L. Contaminação de equipamentos e superfícies de unidades de terapia intensiva de uma maternidade pública por Staphylococcus coagulase negativa. Revista de Patologia Tropical, v. 42, n.4, p.387-394, 2013.

MURRAY, P. R.; ROSENTHAL, K. S.; PFALLER, M. A. Microbiología médica. 7aa ed. Madrid: S.A. Elsevier España, 2013. 888p.

OLAECHEA, P. M.; ÁlVAREZ-LERMA, F.; PALOMAR, M.; INSAUSTI, J.; LÓPEZPUEYO, M. J.; MARTÍNEZ-PELLÚS, A.; CANTÓN, M. L.; Grupo ENVIN-HELICS. Impacto de la bacteriemia primaria y relacionada con cateter intravascular causada por Staphylococcus coagulasa negativo en pacientes críticos. Medicina Intensiva, v. 35, n. 4, p. 217-225, 2011.

OLIVEIRA, A. C.; DE PAULA, A. O. Descolonização de portadores de Staphylococcus aureus: indicações, vantagens e limitações. Texto \& Contexto Enfermagem, v. 21, n. 2, p. 448-457, 2012.

OLIVEIRA, A. C.; SILVA, M. D. M. Caracterização epidemiológica dos microrganismos presentes em jalecos dos profissionais de saúde. Revista Eletrônica de Enfermagem, v. 15, n. 1, p. 80-87, 2013.

OLIVEIRA, I. S. D.; LIMA, C. M. Análise do uso racional de antimicrobianos do hospital público da zona norte de Aracaju. Cadernos de Graduação - Ciências Biológicas e da Saúde, v. 12, n.12, 2010.

OPLUSTIL, C. P.; ZOCOLI, C. M.; TOBOUTI, N. R. Procedimentos básicos em microbiologia clínica. 3. ed. - São Paulo: Sarvier, 2010. 530p.

PEREIRA, E. P. L.; CUNHA, M. L. R. S. Avaliação da colonização nasal por Staphylococcus spp. resistente à oxacilina em alunos de enfermagem. Jornal Brasileiro de Patologia e Medicina Laboratorial, v. 45, n. 5, p. 361-369, 2009.

RABELO, M. A. Estudo epidemiológico, genético e de susceptibilidade em Staphylococcus spp. de amostras de pacientes e profissionais de saúde de hospital universitário de Pernambuco. 2012. Dissertação (Mestrado em Medicina Tropical). Programa de Pós-Graduação em Medicina Tropical, Centro de Ciências da Saúde, Universidade Federal de Pernambuco. Recife, 2012.

RIBEIRO, I. F.; SILVA, S. F. R.; RIBEIRO, T. R.; ROCHA, M. M. N. P.; STOLP, A. M. V. Identificação de Staphylococcus aureus e Staphylococcus aureus resistente à 
meticilina em estudantes universitários. Revista de Ciências Farmacêuticas Básica e Aplicada, v. 35, n. 2, p. 301-304, 2014.

ROSA, J. O.; MOURA, J. P.; PALOS, M. A. P.; GIR, E.; REIS, C.; KIPNIS, A.; CANINI, S. R. M. S.; BELISSIMO-RODRIGUES, F.; PIMENTA, F. C. Detecção do gene mecA em estafilococos coagulase negativa resistentes à oxacilina isolados da saliva de profissionais da enfermagem. Revista Sociedade Brasileira Medicina Tropical, n. 42, v. 4, p. 398-403, 2009.

SANTOS, N. Q. A resistência bacteriana no contexto da infecção hospitalar. Texto \& Contexto Enfermagem, v. 13, p. 64-70, 2004.

SHORE, A. C; COLEMAN, D. C. Staphylococcal Cassette Chromosome mec: Recent Advances and New Insights. International Journal of Medical Microbiology, v. 303, n. 6, p. 350-359, 2013.

SOUZA, A. G. Caracterização fenotípica e molecular de amostras de Staphylococcus coagulase negativo isoladas de infecções da corrente sanguínea de pacientes de dois hospitais gerais da cidade de São Paulo. 2009. 88 f. Dissertação. Programa de pós-graduação em infectologia, Universidade Federal de São Paulo. São Paulo, 2009.

VIANA, A. P. P.; SOARES, R. S.; CASTRO, A. R. L.; KLUCZYNIK, C. E. N.; CATÃO, R. M. R. Incidência bacteriana em hemoculturas de recém-nascidos e perfil de suscetibilidade frente aos antimicrobianos. Revista de Biologia e Farmácia, v. 05, n. 01, 2011. 